\title{
Validation of the Hamilton Anxiety Rating Scale and State Trait Anxiety Inventory A and B in Arabic among the Lebanese population
} \author{
Christiane Abi Elias Hallit ${ }^{\mathrm{c}}$, Pascale Salameh ${ }^{\mathrm{b}, \mathrm{h}, \mathrm{i}}$ \\ ${ }^{a}$ Faculty of Medicine and Medical Sciences, Holy Spirit University of Kaslik (USEK), Jounieh, Lebanon \\ ${ }^{\mathrm{b}}$ INSPECT-LB: Institut National de Santé Publique, Epidémiologie Clinique et Toxicologie, Beirut, Lebanon \\ ${ }^{\mathrm{c}}$ Psychiatric Hospital of the Cross, Jal Eddib, Lebanon \\ ${ }^{\mathrm{d}}$ School of Pharmacy, Lebanese International University, Beirut, Lebanon \\ ${ }^{\mathrm{e}}$ Faculty of Philosophy and Human Sciences, Holy Spirit University, Kaslik, Lebanon \\ ${ }^{\mathrm{f}}$ Faculty of Pedagogy, Lebanese University, Beirut, Lebanon \\ ${ }^{\mathrm{g}}$ Faculty of Pedagogy, Université de la Sainte Famille, Batroun, Lebanon \\ ${ }^{\mathrm{h}}$ Faculty of Pharmacy, Lebanese University, Beirut, Lebanon \\ ${ }^{\mathrm{i}}$ Faculty of Medicine, Lebanese University, Beirut, Lebanon
}

Souheil Hallit ${ }^{\mathrm{a}, \mathrm{b}, *, 1}$, Chadia Haddad ${ }^{\mathrm{a}, 1}$, Rabih Hallit ${ }^{\mathrm{a}}$, Marwan Akel $^{\mathrm{b}, \mathrm{d}}$, Sahar Obeid $^{\mathrm{a}, \mathrm{e}, \mathrm{f}}$ Georges Haddad $^{\mathrm{a}, \mathrm{c}}$, Michel Soufia ${ }^{\mathrm{a}}$, Wael Khansa ${ }^{\mathrm{a}}$, Rony Khoury ${ }^{\mathrm{a}}$, Nelly Kheir ${ }^{\mathrm{g}}$,

A R T I C L E I N F O

A B S T R A C T

Keywords:

Anxiety

HAM-A

STAI-A

STAI-B

Arabic

Validation
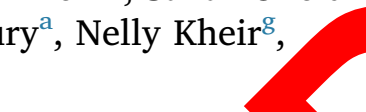
patient's anxiety. Anxiety can denote things such as "a mental state ... a drive ... a response to a particular situation ... a personality trait ... and a psychiatric disorder". ${ }^{10}$ Nonetheless it was one of the first anxiety rating scales to be published, the HAM-A remains widely used by clinicians. ${ }^{9}$ It was originally published by Max Hamilton in 1959. For clinical purposes, only severe or inadequate anxiety is attended to. This scale is considered a "clinical rating" of the range of anxiety, and is proposed for individuals that are "already diagnosed with anxiety neurosis". ${ }^{11}$

As for the State-Trait Anxiety Inventory (STAI), it measures two types of anxiety - state anxiety, or anxiety about an event, and trait anxiety, or anxiety level as a personal characteristic; and used in diagnoses, in both clinical and other medical settings. ${ }^{6}$ The advantage of this scale is that could be applied towards assessing different types of anxiety. This was a new development because all other questionnaires focused on one type of anxiety at the time. ${ }^{12}$

Appropriate, validated and translated scales are needed to explain and predict anxiety in each country due to the influence of linguistic and cultural differences. The use of the validated scale in different languages, to assess the severity of the anxiety symptoms, demands two concepts: (1) maintaining a level of psychometric properties comparable to the original version and (2) adapting the scale to the cultural of the country. The validated and translated scale with a good reliability, will be helpful in clinical settings and research. ${ }^{13}$

Therefore, it is essential to validate the Arabic version of the STAI and HAM-A scales for cultural adaptation among the Lebanese population in order to measure the severity of anxiety. Our aim in this study was to translate the HAM-A, STAI-A and B scales to Arabic, linguistically validate them for use in a representative sample of the Lebanese population, and check the reliability of these Arabic versions.

\section{Methods}

\subsection{Study design and sampling (Sample 1)}

This study is cross-sectional, conducted between No bu 17 and March 2018, which enrolled 1332 comp pants using a proportionate random Mohafazat (Beirut, Mount Lebanon, No Mohafaza is divided into Caza (straty two village. re ranumly selected from the list of villages $r$ ded b e Cen gency of Statistics in Lebanon. Patients we do selected from each village. All participants above 18 vears s vere eli to participate. Excluded where the patien sych rob mental retardation, dementia or who ed to the qu aire. Data collection was performed thr perso intervieu with participants by trained, study indeper

\subsection{Ethical approval}

The Psychiatric Hospital of the Cross Ethics and Research Committee, in compliance with the Hospital's Regulatory Research Protocol, approved this study protocol (HPC-009-2018) based on the fact that the autonomy and confidentiality of participants were respected and since it was an observational study, no harm will be prompted to them. The purpose and requirement of the study was informed to each patients. Consent was obtained as written approval on the ethical consent form.

\subsection{Minimal sample size calculation}

Comrey and Lee suggested that a minimum of 10 observations per variable is necessary in order to avoid computational difficulties. ${ }^{14}$ Since the STAI-A and B scales questionnaire contains 40 questions (20 questions for each scale), a minimal sample of 400patients was needed to conduct an exploratory factor analysis.
Table 1

Sociodemographic characteristics of the sample population.

\begin{tabular}{|c|c|}
\hline & Frequency (\%) \\
\hline \multicolumn{2}{|l|}{ Gender } \\
\hline Male & $480(36.1 \%)$ \\
\hline Female & $848(63.9 \%)$ \\
\hline \multicolumn{2}{|l|}{ Education level } \\
\hline Illiterate & $13(1.0 \%)$ \\
\hline Primary & $21(1.6 \%)$ \\
\hline Complementary & $64(4.8 \%)$ \\
\hline Secondary & $224(16.9 \%)$ \\
\hline University & $883(66.7 \%)$ \\
\hline Higher education & $119(9.0 \%)$ \\
\hline \multicolumn{2}{|l|}{ Employment status } \\
\hline Unemployed & $917(68.8 \%)$ \\
\hline Employed & $415(31.2 \%)$ \\
\hline \multicolumn{2}{|l|}{ Socioeconomic status } \\
\hline$<1000 \$$ & $926(73.5 \%)$ \\
\hline $1000-2000 \$$ & $251(19.9 \%)$ \\
\hline$>2000 \$$ & $83(6.6 \%)$ \\
\hline \multicolumn{2}{|l|}{ Marital status } \\
\hline Single & $963(72.6 \%)$ \\
\hline Marrie & $334(25.2 \%)$ \\
\hline & $13(1.0 \%)$ \\
\hline & $17(1.3 \%)$ \\
\hline & $68(5.1 \%)$ \\
\hline & $162(12.2 \%)$ \\
\hline & $279(21.0 \%)$ \\
\hline & $335(25.3 \%)$ \\
\hline & $482(36.3 \%)$ \\
\hline & $96(7.3 \%)$ \\
\hline & $127(9.6 \%)$ \\
\hline Complementary & $302(22.9 \%)$ \\
\hline Secondary & $393(29.8 \%)$ \\
\hline University & $402(30.5 \%)$ \\
\hline \multicolumn{2}{|l|}{ Residence } \\
\hline Alone & $95(7.2 \%)$ \\
\hline With others & $1217(92.8 \%)$ \\
\hline \multicolumn{2}{|c|}{ Personal history of medical illness } \\
\hline Yes & $157(11.8 \%)$ \\
\hline No & $1174(88.2 \%)$ \\
\hline \multicolumn{2}{|c|}{ Family history of psychiatric illness } \\
\hline Yes & $69(5.2 \%)$ \\
\hline \multirow[t]{2}{*}{ No } & $1262(94.8 \%)$ \\
\hline & Mean \pm SD \\
\hline Age (in years) & $28.08 \pm 16.79$ \\
\hline Number of kids & $1.15 \pm 1.72$ \\
\hline
\end{tabular}

\subsection{Questionnaire}

The questionnaire used during the interviewed was in Arabic, the native language of Lebanon. A trained staff was in charge of collecting the data, via a personal interview with each participant. This person was independent of this study. The first part assessed the sociodemographic characteristics of the included population (age, gender, educational level of the patient and his parents, marital status, socioeconomic level, alcohol consumption, family history of psychiatric diseases). The socioeconomic level, defined as the family monthly income, was divided into 3 categories: low $(<1000$ USD, intermediate (1000-2000 USD) and high (2000 USD). Also, we asked the patient if he got a physician diagnosis of anxiety and was taking a medication to treat it. The other parts comprised the different scales used in this study as follows:

\subsection{Hamilton anxiety scale (HAM-A)}

The HAM-A ${ }^{15}$ entails 14 items, each categorized by a series of symptoms, and measures mental agitation and psychological distress, as 
Table 2

Promax rotated matrix of HAMA scale factors, Sample 1, and confirmatory factor analysis parameters, Sample 2 (italics).

\begin{tabular}{llll}
\hline & Items & Factor 1 & Factor 2 \\
\hline Genitourinary symptoms & 12 & $0.881 / 0.401$ & \\
Cardiovascular symptoms & 9 & $0.821 / 0.584$ & \\
Autonomic symptoms & 13 & $0.800 / 0.742$ & \\
Respiratory symptoms & 10 & $0.774 / 0.633$ & \\
Gastrointestinal symptoms & 11 & $0.721 / 0.597$ & \\
Somatic (sensory) & 8 & $0.667 / 0.810$ & \\
Behavior at interview & 14 & $0.643 / 0.376$ & \\
Somatic (muscular) & 7 & $0.551 / 0.694$ & \\
Tension & 2 & & $0.910 / 0.916$ \\
Anxious mood & 1 & & $0.903 / 0.842$ \\
Depressed mood & 6 & & $0.648 / 0.942$ \\
Insomnia & 4 & & $0.529 / 0.521$ \\
Fears & 3 & & $0.486 / 0.596$ \\
Intellectual & 5 & &
\end{tabular}

Factor 1 = Somatic items; Factor $2=$ Psychic/Psychological items.

Cronbach's alphas: factor $1=0.898$; factor $2=0.853$; total scale $=0.921$.

Percentage of variance explained: $58.51 \%$.

Kaiser-Meyer-Olkin $(\mathrm{KMO})=0.947$.

Bartlett's test of sphericity $=\mathrm{p}<0.001$.

well as anxiety-related physical complaints. The responses on the scale were measured on a 5-point Likert scale: 0 (symptoms not present), 1 (mild symptoms), 2 (moderate symptoms), 3 (severe symptoms) and 4 (very severe symptoms). The total score was calculated by summation of the 14 items.

\subsection{Forward translation into Arabic}

A single bilingual translator, Arabic native fluent in English, aware of the concepts of the three anxiety scales translated the English versions of the scales into Arabic. An expert committee, composed of health care professionals (psychiatrists and psychologists), a language professional and the original translator, had reviewed and revised the translated questionnaire in order to check for idiomatic and conceptual equivalence of the Arabic translated version. ${ }^{17-20}$

\subsection{Back translation into English}

A native English speaker translator person, fluent in Arabic, had back translated the Arabic versions of the three scales into the English language. The translator was unaware with the concepts of the anxiety scales and the original English ${ }^{17,18}$ The expert committee compared the back-translated of the questionnaire with the original English one, $i$ aer to che $r$ inconsistencies and to solve any discrepancies bo n the vers s. All ambiguities disappeared after repeat the $p_{1}$ of for d-back translation. ${ }^{17-22}$

2.9. Sample 2

We co cro ctional study in May 2018 on a sample of anese nts nding psychology clinic that enrolled 155 particip s. To en validity of the results, the scales was on and sample sample 2), independent from the first one. Patu estionnaire through a face-to-face interview.

\subsection{State-Trait Anxiety Inventory (STAI) A and B}

It is composed of 40 questions that measure two types of an state anxiety, or anxiety about an event (STAI-A), and tr anxiety level as a personal characteristic (STAI-B). The a 4-point Likert scale, with 1 (not at all), 2 (somewhat), 3 (m and 4 (very much so) for STAI-A and 1 (almost (often) and 4 (almost always) for STAI-B. High correlated with higher levels of anxiety. ${ }^{16}$

Table 3

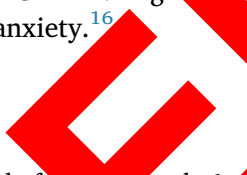

Promax rotated matrix of STAI-A scale fa ample 1 confirmatory factor analysis parameters, Sample 2 (italics).

\begin{tabular}{|c|c|c|c|c|}
\hline & Items & Factor 1 & Factor 2 & Factor 3 \\
\hline I feel steady & 19 & $0.795 / 0.497$ & & \\
\hline I feel pleasant & 20 & $0.713 / 0.635$ & & \\
\hline I feel content & 16 & $0.696 / 0.609$ & & \\
\hline I feel self-confident & 11 & $0.659 / 0.557$ & & \\
\hline I feel satisfied & 8 & $0.597 / 0.668$ & & \\
\hline I feel at ease & 5 & $0.582 / 0.612$ & & \\
\hline I feel comfortable & 10 & $0.538 / 0.580$ & & \\
\hline I am relaxed & 15 & $0.467 / 0.570$ & & \\
\hline I am tense & 3 & & $0.779 / 0.694$ & \\
\hline I feel strained & 4 & & $0.705 / 0.544$ & \\
\hline I feel nervous & 12 & & $0.647 / 0.833$ & \\
\hline I am worried & 17 & & $0.601 / 0.747$ & \\
\hline I feel upset & 6 & & $0.587 / 0.765$ & \\
\hline I feel calm & 1 & & $0.510 / 0.609$ & \\
\hline I feel secure & 2 & & $0.508 / 0.488$ & \\
\hline I feel frightened & 9 & & & $0.721 / 0.564$ \\
\hline I am jittery & 13 & & & $0.706 / 0.583$ \\
\hline I feel indecisive & 14 & & & $0.635 / 0.671$ \\
\hline I am presently worrying over possible misfortunes & 7 & & & $0.586 / 0.533$ \\
\hline I feel confused & 18 & & & $0.449 / 0.283$ \\
\hline
\end{tabular}

Cronbach's alphas: factor $1=0.852$; factor $2=0.855$; factor $3=0.780$; total scale $=0.928$.

Percentage of variance explained: $56.04 \%$.

Kaiser-Meyer-Olkin (KMO) $=0.953$.

Bartlett's test of sphericity $=\mathrm{p}<0.001$. 
Table 4

Promax rotated matrix of STAI-B scale factors, Sample 1, and confirmatory factor analysis parameters, Sample 2 (italics).

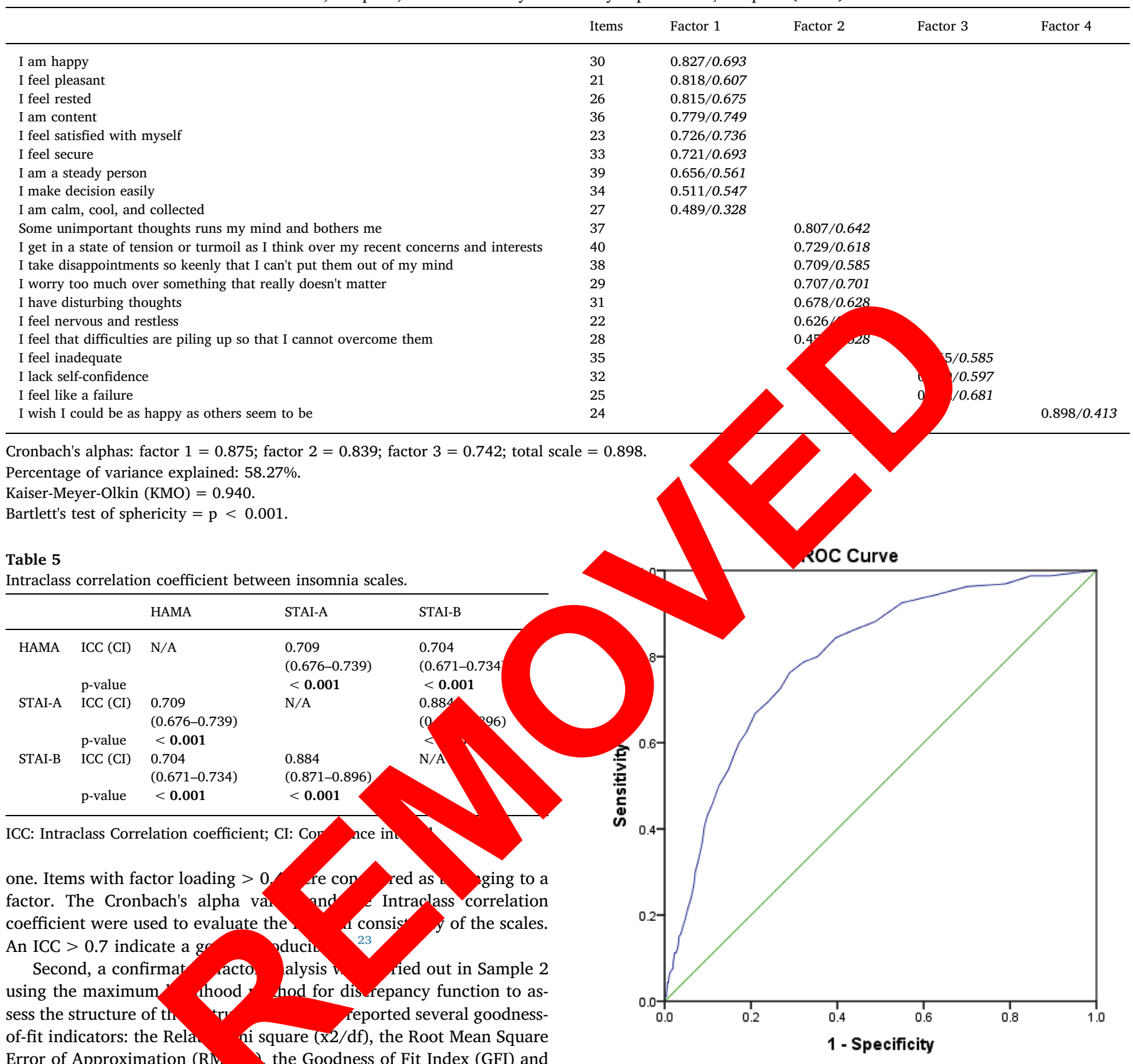
Error of Approximation (RM the Goodness of Fit Index (GFI) and the Adjusted Goodness of Fit $\mathrm{h}$ (AGFI). The value of $\mathrm{x} 2$ divided by the degrees of freedom (x2/df) has a low sensitivity to sample size and may be used as an index of goodness of fit (cut-off values: $<2-5$ ). The RMSEA tests the fit of the model to the covariance matrix. As a guideline, values of $<0.05$ indicate a close fit and values below 0.11 an acceptable fit. The GFI and AGFI are chi-square-based calculations independent of degrees of freedom. The recommended thresholds for acceptable values are $\geq 0.90$. $^{24}$

\section{Results}

The sociodemographic characteristics of the participants are summarized in Table 1 . The results showed that the mean age of the participants was $28.08 \pm 16.79$ years, with $63.9 \%$ females. The majority $(75.7 \%)$ had a university level of education, unemployed $(68.8 \%)$, single $(72.6 \%)$, with a low monthly income (<1000 USD) $(73.5 \%)$.

Fig. 1. ROC curve of the HAM-A scale. Patients diagnosed with anxiety by the physician were analyzed. Area under the curve $=0.795$ [0.760-0.830] $(\mathrm{P}<0.001)$; at value $=11.50, \mathrm{Se}=76.3 \%$ and $\mathrm{Sp}=71.1 \%$.

Only $5.2 \%$ of the participants had a family history of psychiatric illnesses, whereas $11.8 \%$ had a history of medical illness.

\subsection{Factor analysis}

Three factor analyses for the anxiety scales were run over the whole sample $(\mathrm{N}=1332)$. All of the HAM-A, STAI-A and STAI-B items could be extracted from the list. All items from all the scales did not overcorrelate to each other $(r>0.9)$, did not have a low loading on factors $(<0.3)$ or a low communality (a communality is the extent to which an item correlates with all other items) $(<0.3)$. The number of loading factors, KMO and Bartlett's test of sphericity values and the Cronbach alpha values for each scale are summarized in Table 2 (HAM-A), Table 3 


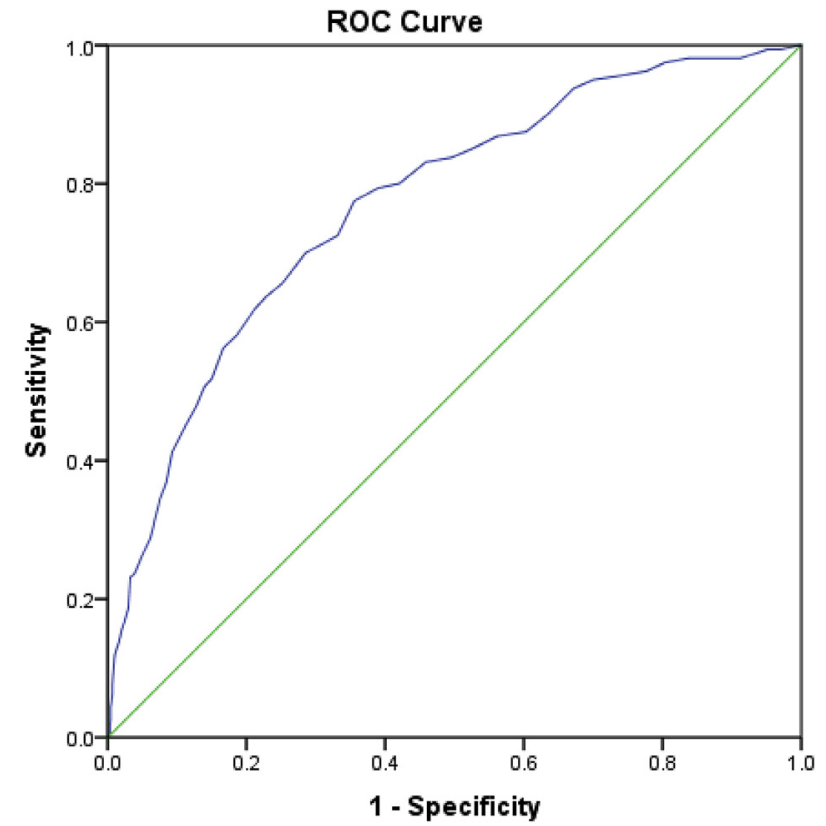

Fig. 2. ROC curve of the STAI-A scale. Patients diagnosed with anxiety by the physician were analyzed. Area under the curve $=0.771$ [0.732-0.810] $(\mathrm{P}<0.001)$; at value $=42.50$, $\mathrm{Se}=71.3 \%$ and $\mathrm{Sp}=69.1 \%$.
Square $=405.995$ and Degrees of Freedom $=167$, which gave an $\mathrm{x} 2$ / $\mathrm{df}=2.43$. For non-centrality fit indices, the Steiger-Lind RMSEA was 0.094 [0.082-0.108]. Moreover, the Joreskog GFI equaled 0.789 and AGFI equaled 0.734 .

For the STAI-B scale the Maximum Likelihood ChiSquare $=344.374$ and Degrees of Freedom $=165$, which gave an $\mathrm{x} 2$ / $\mathrm{df}=2.08$. For non-centrality fit indices, the Steiger-Lind RMSEA was 0.087 [0.074-0.099]. Moreover, the Joreskog GFI equaled 0.806 and AGFI equaled 0.753 .

\subsection{Intraclass correlation coefficient between insomnia scales}

A significantly high ICC was found between the HAM-A, STAI-A (ICC $=0.709$ ) and STAI-B (0.704). In addition, a significantly high ICC was found between the STAI-A and B scales $($ ICC $=0.884)$ (Table 5).

\subsection{ROC curves}

The receiver operating cteristic (1) curve of the anxiety score as calculated he Hh scale, paring patients with a physician diagnos anxiety to th es, showed that the area under the cur as hi $0.790 \quad 00-0.830](\mathrm{P}<0.001)$; at value $=11.50$, cenc $\quad$ y was $76.3 \%$ and the specificity was $71.1 \%$ (Fig. 1). The R urve
scale, comp a patio $b$ hy ones, wed than he area under the curve was high $=0.771$ [0. 10] $<0.001$ ); at value $=42.50$, the sensitivity was $71.3 \%$ an ificity was $69.1 \%$ (Fig. 2).

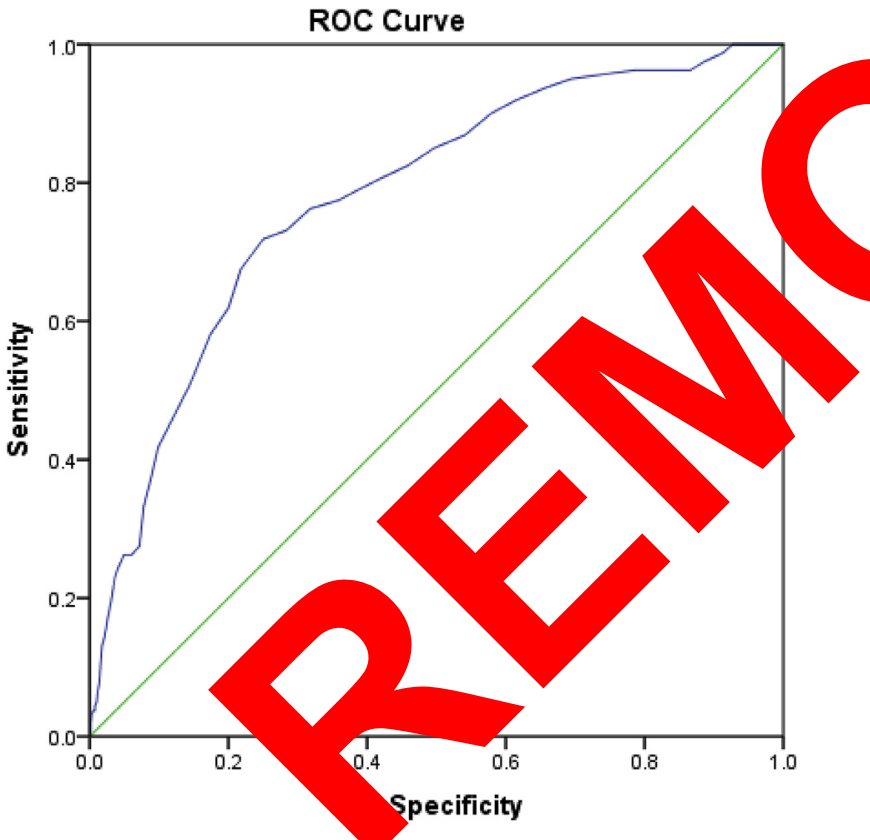

Fig. 3. ROC curve of the STAI-B scale. Patients diagnosed with anxiety by the physician were analyzed. Area under the curve $=0.779$ [0.741-0.817] $(\mathrm{P}<0.001)$; at value $=45.50, \mathrm{Se}=73.1 \%$ and $\mathrm{Sp}=71.6 \%$.

(STAI-A) and Table 4 (STAI-B) respectively.

\subsection{Sample 2}

A confirmatory factor analysis was run on sample 2, using the structure obtained in Sample 1. The following results was obtained: for the HAMA scale, the Maximum Likelihood Chi-Square $=152.509$ and Degrees of Freedom $=76$, which gave an $\mathrm{x} 2 / \mathrm{df}=2.01$. For non-centrality fit indices, the Steiger-Lind RMSEA was 0.077 [0.057-0.096]. Moreover, the Joreskog GFI equaled 0.98 and AGFI equaled 0.93 .

For the STAI-A scale the Maximum Likelihood Chi- he ROC an of the anxiety score as calculated by the STAI-B sca omparing patients with a physician diagnosis of anxiety to heal ones, showed that the area under the curve was high $=0.779$ [0.74.817] $(\mathrm{P}<0.001)$; at value $=42.50$, the sensitivity was 73 and the specificity was $71.6 \%$ (Fig. 3).

3.5. Face validity

When comparing the scales scores between patients with and without physician diagnosis of anxiety, the results showed that significantly higher means HAM-A, STAI-A and B scores were found in patients with a physician diagnosis of anxiety compared to those without the diagnosis ( $\mathrm{p}<0.001$ for the three scales) (Fig. 4).

\section{Discussion}

The Arabic versions of the HAM-A, STAI-A and B scales was validated in the current study for use among the Lebanese population. The results showed a high reliability and validity of these scales that provide an initial evidence that could be used as a screening instrument for anxiety in Lebanon. The translation process was conducted by independent translators, the point that decreases the subjectivity of the scales' adaptation. A comparison of the fit of the form obtained through the confirmatory factor analysis revealed that the 2 factors for HAM-A, 3 factors for STAI-A and 4 factors for STAI-B had high fit indices.

\subsection{Validation of the scales}

The construct validity of the STAI-A and B scales were adequate because items converged over three factors for STAI-A and 4 factors for STAI-B, with adequate factor loadings for all items. The internal consistency of both scales was similar to that obtained in other translated versions for state (Cronbach alpha of 0.93 for state and 0.92 for trait scales in Greece ${ }^{25}$ and 0.93 to 0.95 for state and 0.91 to 0.93 for trait anxiety in a group of Lebanese and American students ${ }^{26}$ ), but lower to that obtained in. The results of the Arabic version of the STAI validity and reliability, had been similar to the psychometric properties 


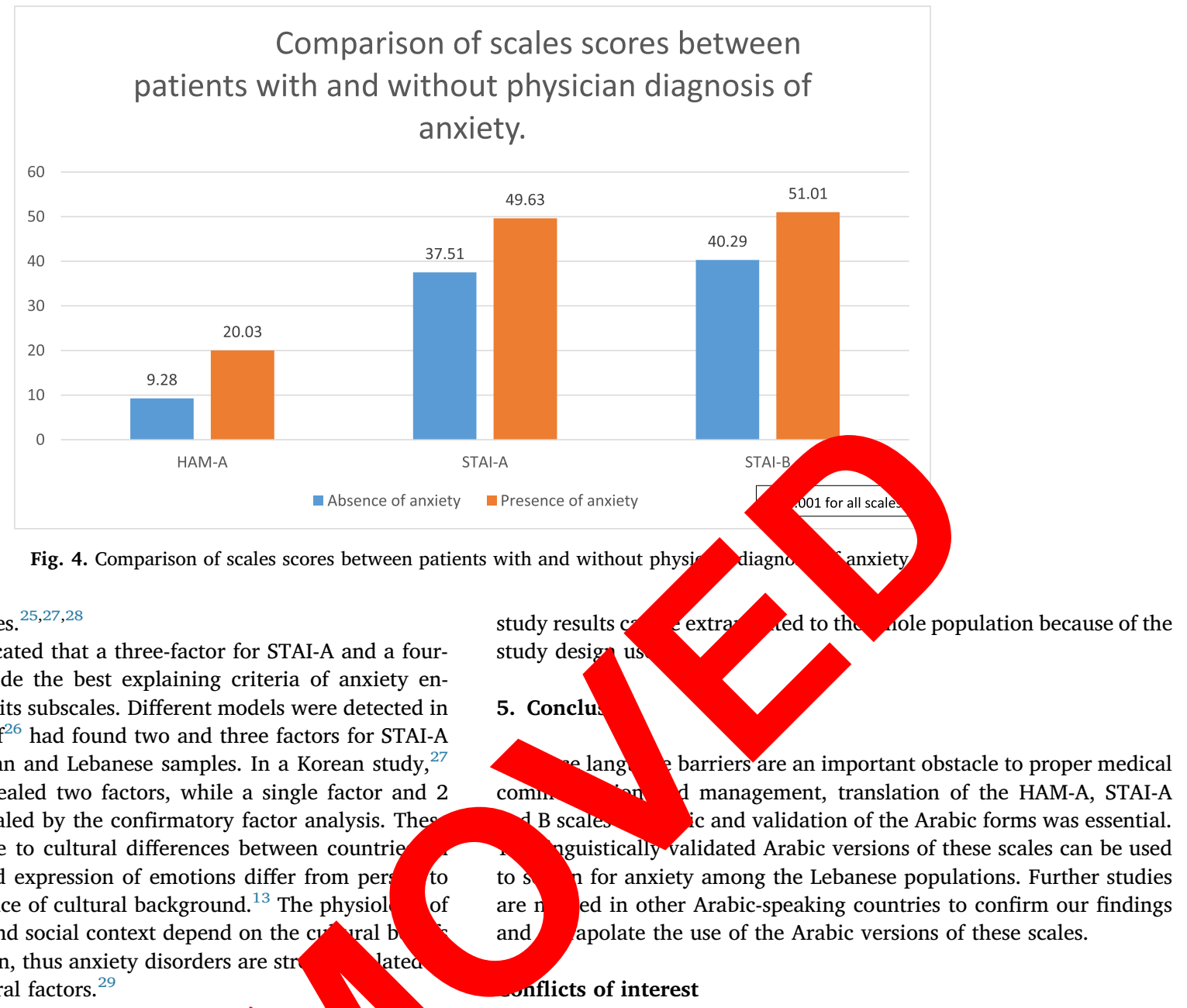

reported by other studies. ${ }^{25,27,28}$

Factor analysis indicated that a three-factor for STAI-A and a fourfactor for STAI-B provide the best explaining criteria of anxiety enclosing the state and traits subscales. Different models were detected in other studies; Abdullatif ${ }^{26}$ had found two and three factors for STAI-A and STAI-B for American and Lebanese samples. In a Korean study, ${ }^{27}$ factor analysis had revealed two factors, while a single factor and 2 factor models was revealed by the confirmatory factor analysis. The variations might be due to cultural differences between countrie fact, the experience and expression of emotions differ from per to other due to the influence of cultural background. ${ }^{13}$ The physiol of the illness syndromes and social context depend on the cy ral b that varied across person, thus anxiety disorders are str racial, ethnic and cultural factors. ${ }^{29}$

The construct validity of the HAM-A scale cause items converged over two factors Psychologic), with adequate factor loadingo results obtained from previous researc consistency of the HAM-A scale was this scale's items are able to scr population in an excellent way.

\subsection{Validity}

The construct va addressed by calculating study is the first to our validation process of these sa We obtained good areas under the curves, sensitivities and specificries for all three scales. These good results may be due to the fact that we compared used the physician diagnosis variable to compare between individuals with anxiety and those without.

\subsection{Limitations}

The current study has several limitations. An information bias could have occurred since the patients might not have understood the questions well or over/underestimated the answers to some questions. A selection bias is possible because of the refusal rate. The levels of experiential and expressive anxiety are gender different thus our results could be affected by the unequal number of males and females. In addition, the majority of the participants were young according to the mean age, therefore, future studies should be targeted towards older adults. Despite these limitations, the sample size is acceptable and the
The authors have nothing to disclose.

\section{Funding sources}

None.

\section{Appendix A. Supplementary data}

Supplementary data to this article can be found online at https:// doi.org/10.1016/j.cegh.2019.02.002.

\section{References}

1. Spielberger CD. Anxiety as an emotional state. In: Spielberger CD, ed. Anxiety: Current Trends in Theory and Research. vol. 1. New York: Academic Press; 1972:23-49https:// doi.org/10.1016/B978-0-12-657401-2.50009-5.

2. Ollendick Thomas, King Neville, Yule William. International Handbook of Phobic and Anxiety Disorders in Children and Adolescents. New York: Plenum Press; 1994.

3. Julian LJ. Measures of anxiety: state-trait anxiety inventory (STAI), Beck anxiety inventory (BAI), and Hospital anxiety and Depression scale-anxiety (HADS-A). Arthritis Care Res. 2011;63(S11):S467-S472.

4. Spielberger CD. Assessment of state and trait anxiety: conceptual and methodological issues. South Psychol. 1985;2(4):6-16.

5. Spielberger CD. State-Trait Anxiety Inventory: Bibliography. 2nd ed. Palo Alto: Consulting Psychologists Press; 1989.

6. Spielberger CD, Gorsuch RL, Lushene R, Vagg PR, Jacobs GA. Manual for the StateTrait Anxiety Inventory. Palo Alto, CA: Consulting Psychologists Press; 1983

7. Bahammam MA. Validity and reliability of an Arabic version of the state-trait anxiety inventory in a Saudi dental setting. Saudi Med J. 2016;37(6):668.

8. Clark DB, Donovan JE. Reliability and validity of the Hamilton anxiety rating scale in an adolescent sample. $J$ Am Acad Child Adolesc Psychiatry. 1994;33(3):354-360.

9. Maier W, Buller R, Philipp M, Heuser I. The Hamilton Anxiety Scale: reliability, validity and sensitivity to change in anxiety and depressive disorders. $J$ Affect Disord. 1988;14(1):61-68 
10. McDowell I. Measuring Health: A Guide to Rating Scales and Questionnaires. USA: Oxford University Press; 2006

11. Hamilton M. The assessment of anxiety states by rating. Br J Med Psychol 1959;32(1):50-55.

12. Tilton S. Review of the state-trait anxiety inventory (STAI). New Notes. 2008;48(2):1-3.

13. Hofmann SG, Hinton DE. Cross-cultural aspects of anxiety disorders. Curr Psychiatr Rep. 2014;16(6):450.

14. Comrey AL, Lee HB. A First Course in Factor analysis. Psychology Press; 2013.

15. Hamilton M. The assessment of anxiety states by rating. Br J Med Psychol 1959;32(1):50-55.

16. Spielberger CD, Sydeman SJ. State-Trait Anxiety Inventory and State-Trait Anger expression inventory. In: Maruish ME, ed. The Use of Psychological Testing for Treatment Planning and Outcome Assessment. Hillsdale, NJ, USA: L. Erlbaum Associates; 1994:292-321https://doi.org/10.1002/9780470479216.corpsy0943.

17. Beaton DE, Bombardier C, Guillemin F, Ferraz MB. Guidelines for the process of cross-cultural adaptation of self-report measures. Spine (Phila Pa 1976). 2000;25(24):3186-3191.

18. Beaton D, Bombardier C, Guillemin F, Ferraz MB. Recommendations for the CrossCultural Adaptation of Health Status Measures. New York: American Academy of Orthopaedic Surgeons; 2002:1-9.

19. Al-Bannay H, Jarus T, Jongbloed L, Yazigi M, Dean E. Culture as a variable in health research: perspectives and caveats. Health Promot Int 2014;29(3):549-557.

20. Gutiérrez-Sánchez D, Leiva-Santos JP, Sánchez-Hernández R, Hernández-Marrero D, Cuesta-Vargas AI. Spanish modified version of the palliative care outcome scalesymptoms renal: cross-cultural adaptation and validation. BMC Nephrol. 2016 Nov $18 ; 17(1): 180$

21. Maneesriwongul W, Dixon JK. Instrument translation process: a methods review. $J$
Adv Nurs. 2004;48(2):175-186.

22. Nejjari C, El Fakir S, Bendahhou K, et al. Translation and validation of European organization for research and treatment of cancer quality of life Questionnaire -C30 into Moroccan version for cancer patients in Morocco. BMC Res Notes. 2014;7:228.

23. Terwee CB, Bot SD, de Boer MR, et al. Quality criteria were proposed for measurement properties of health status questionnaires. J Clin Epidemiol. 2007;60(1):34-42.

24. Marsh Herb, Hau KT, Wen Z. In search of golden rules: comment on hypothesistesting approaches to setting cutoff values for fit indexes and dangers in overgeneralizing Hu and Bentler's (1999) findings. Struct Equ Model A Multidiscip j. January 2004;11:320-341.

25. Fountoulakis KN, Papadopoulou M, Kleanthous S, et al. Reliability and psychometric properties of the Greek translation of the State-Trait Anxiety Inventory form Y: preliminary data. Ann Gen Psychiatr. 2006;5:2.

26. Abdullatif QA. Adaptation of the State Trait anxiety Inventory in Arabic: A Comparison with the American STAI USA: University of South Florida; 2004 http://www. scholarcommons.usf.edu/cgi/viewcontent.cgi article $=1932 \&$ context $=$ etd. Available from:

27. Bieling PJ, Antony MM, Swinson RP. The State-Trait Anxiety Inventory, Trait version: structure and content re-examined. Behav Res Ther. 1998 Jul-Aug;36(78):777-788.

28. Barnes LL, Harp D, Jung WS. Reliab lization of scores on the Spielberger state-trait anxiety inventory. Edv 02;62(4):603-618. https://doi. org $/ 10.1177 / 0013164402062$

29. Hinton DE Multicultural che Anxiety. 2012;29(1):1-3

30. Hofmann SG, Anu As? social anxiety diso

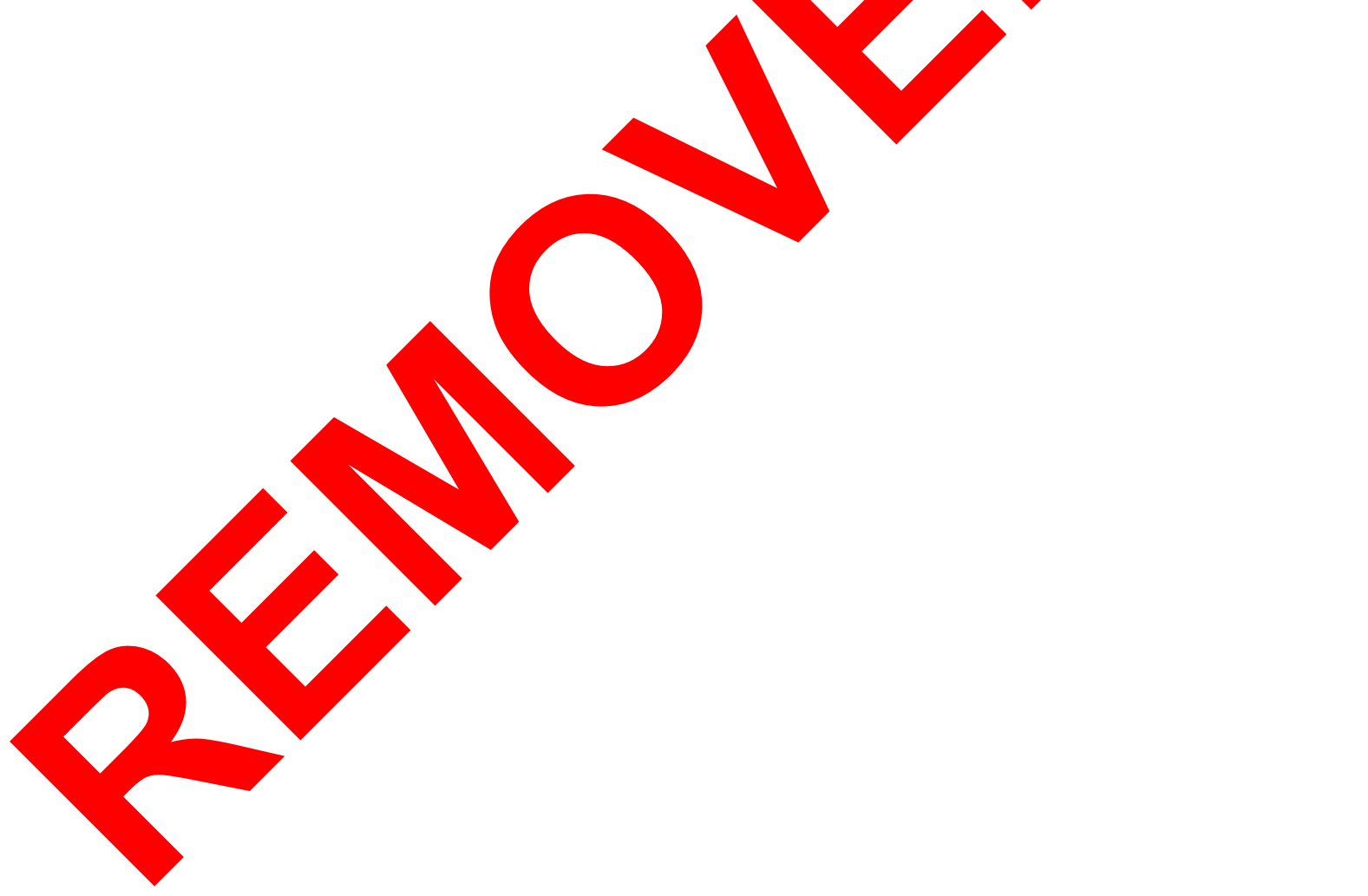

